\title{
Eficiência de fungos ectomicorrízicos na absorção de fósforo e na promoção do crescimento de eucalipto
}

\author{
Luiz Afonso Borges de Souza(1), Germano Nunes Silva Filho(1) e Vetúria Lopes de Oliveira(1)
}

\begin{abstract}
(1)Universidade Federal de Santa Catarina, Centro de Ciências Biológicas, Dep. de Microbiologia e Parasitologia, Caixa Postal 476 , CEP 88040-900 Florianópolis, SC. E-mail: borges@ccb.ufsc.br, germano@ccb.ufsc.br, veturia@ccb.ufsc.br
\end{abstract}

\begin{abstract}
Resumo - O potencial de fungos ectomicorrízicos na promoção do crescimento das plantas e o controle da micorrização podem alavancar a exploração comercial desses fungos. O objetivo deste trabalho foi avaliar a eficiência de isolados de fungos ectomicorrízicos na colonização radicular, absorção de $\mathrm{P}$ e no crescimento de mudas de Eucalyptus dunnii, sob diferentes doses de fósforo. O inóculo dos isolados fúngicos INRA-Cou (Paxillus involutus), UFSC-Hg93 (Hysterangium gardneri), UFSC-Pt116 (Pisolithus microcarpus) e UFSC-Ch163 (Chondrogaster angustisporus) foi produzido assepticamente numa mistura turfa-vermiculitameio de cultura. As plantas foram cultivadas num substrato de igual composição, suplementado com macro e micronutrientes, e diferentes doses de fósforo: 0, 0,25, 0,50, 1,00, 2,00, 4,00 e 8,00 mg por planta. Os isolados UFSC-Pt116 e UFSC-Ch163 promoveram maior colonização radicular, absorção de $\mathrm{P}$ e crescimento das plantas, medido pela altura e massa de matéria seca. A colonização micorrízica foi inibida pelo aumento das doses de fósforo. As doses de 0,50 e 1,00 mg de P por planta mostraram-se mais adequadas em compatibilizar a colonização radicular com um bom crescimento das mudas. Os isolados UFSC-Ch163 e UFSC-Pt116 são promissores em um programa de micorrização controlada de E. dunnii.
\end{abstract}

Termos para indexação: Eucalyptus dunnii, Pisolithus microcarpus, Chondrogaster angustisporus, ectomicorrizas, inoculação.

\section{Efficiency of ectomycorrhizal fungi on phosporus uptake and eucalypt growth promotion}

\begin{abstract}
The potential of ectomycorrhizal fungi to promote plant growth and the mycorrhization control may contribute to important progress towards the exploitation of these fungi commercially. The objective of this work was to evaluate the efficiency of ectomycorrhizal isolates on root colonization, P uptake and growth of Eucalyptus dunnii seedlings under different levels of phosphorus applied. Inocula of the ectomycorrhizal isolates, INRA-Cou (Paxillus involutus), UFSC-Hg93 (Hysterangium gardneri), UFSC-Pt116 (Pisolithus microcarpus) and UFSC-Ch163 (Chondrogaster angustisporus), were aseptically produced in a peat-vermiculite mixture supplemented with liquid culture medium. Plants were grown in a similar substrate supplemented with macroand micronutrients, under different levels of phosphorus: $0,0.25,0.50,1.00,2.00,4.00$, and $8.00 \mathrm{mg}$ per plant. The isolates UFSC-Pt116 and UFSC-Ch163 promoted higher root colonization, higher P uptake and plant growth, as determined by plant height and dry matter. Levels of 0.50 and $1.00 \mathrm{mg}$ of $\mathrm{P}$ per plant promoted a significant mycorrhizal colonization and an adequate plant growth. The isolates UFSC-Ch163 and UFSC-Pt116 represent a promising alternative for a controlled mycorrhization programme of E. dunnii.
\end{abstract}

Index terms: Eucalyptus dunnii, Pisolithus microcarpus, Chondrogaster angustisporus, ectomycorrhizas, inoculation.

\section{Introdução}

O controle da micorrização consiste na inoculação de fungos específicos do solo em plantas, baseando-se numa relação mutualística entre esses organismos (Garbaye, 1990). O processo tem início com a seleção de isolados fúngicos mais eficientes e culmina com a produção de inoculantes mais adequados à espécie cultivada (Marx et al., 1977; Garbaye, 1990; Rossi et al.,
2002). A relevância dessa estratégia de manejo fica mais evidente ao se constatar que as principais espécies florestais cultivadas no país, Pinus spp. e Eucalyptus spp., são dependentes dessa simbiose para melhor sobrevivência e crescimento (Wilcox, 1990).

A importância da associação ectomicorrízica foi demonstrada nas primeiras tentativas de implantação de espécies vegetais fora de seus habitats naturais, e na 
dificuldade em se estebelecerem áreas de florestamentos em regiões de solos degradados, onde não existiam fungos compatíveis com as espécies introduzidas (Vozzo \& Hacskaylo, 1971; Mikola, 1973).

As ectomicorrizas aumentam a área de absorção radicular, permitindo um melhor aproveitamento de água e nutrientes, como o P, N e K (Glowa et al., 2003; Sawyer et al., 2003). Propiciam, também, maior resistência a estresses hídricos, a temperaturas mais elevadas e acidez do solo, além de possibilitar maior tolerância à presença de substâncias tóxicas no solo e a patógenos do sistema radicular (Marx \& Cordell, 1989; Smith \& Read, 1997). Portanto, contribuem no estabelecimento e no crescimento das plantas, mesmo em solos pobres em nutrientes ou degradados (Marx \& Ruehle, 1988; Marx $\&$ Cordell, 1989).

Os fungos ectomicorrízicos (fECM) têm capacidade diferenciada em colonizar e promover benefícios às espécies vegetais. Em condições específicas, há diferentes respostas, variando em compatibilidade e eficiência (Garbaye, 1990; Sawyer et al., 2003). Haselwandter \& Bowen (1996) sugerem que as melhores respostas são obtidas com a inoculação de fECM selecionados por sua eficiência na melhoria da absorção de nutrientes, em particular para solos de baixa fertilidade.

Os fECM pertencem a diversas famílias dos filos Basidiomycota e Ascomycota e são, portanto, um grupo polifilético (Smith \& Read, 1997). Esse fato permite supor que as diferentes espécies de fECM possam apresentar comportamento variável em relação às suas exigências nutricionais e ambientais. Assim, embora muitos estudos sobre a inoculação desses organismos em plantas venham demonstrando que os efeitos benéficos se manifestam principalmente em condições de baixa fertilidade do solo/substrato, sabe-se que os benefícios nutricionais não são os únicos proporcionados pelos fungos ectomicorrízicos a seus hospedeiros, enfatizando o caráter multifuncional das associações micorrízicas proposto por Newsham et al. (1995).

Assim, estudos visando selecionar isolados de fECM são essenciais a um programa de micorrização controlada, que busque o aumento da produtividade (Alves et al., 2001). Para tanto, é necessário determinar as melhores combinações fungo-hospedeiro e métodos, como substratos mais adequados, doses corretas de nutrientes, teores de umidade, porcentagem de inóculo e, por fim, métodos de produção de inoculantes.

O objetivo deste trabalho foi avaliar a eficiência de isolados de fECM na colonização radicular, absorção de $\mathrm{P}$ e no crescimento de mudas de Eucalyptus dunnii Maiden, sob diferentes doses de fósforo.

\section{Material e Métodos}

Foram estudados quatro isolados de fungos ectomicorrízicos: INRA-Cou, UFSC-Ch163, UFSC-Hg93 e UFSC-Pt116, pertencentes às espécies Paxillus involutus (Batsch) Fr., Chondrogaster angustisporus Giachini, Castellano, Trappe \& Oliveira, Hysterangium gardneri Fischer e Pisolithus microcarpus (Cooke \& Massee) Cunn., respectivamente. O primeiro isolado é proveniente da localidade de Cousserges, sul da França, e os três últimos foram isolados de frutificações coletadas no Estado de Santa Catarina. C. angustisporus é uma nova espécie, encontrada no Brasil e na Austrália, e descrita recentemente por Giachini et al. (2000). As duas outras representam novos relatos no Brasil, de acordo com esses autores.

Esses isolados são mantidos em meio Melin-Norkrans modificado (MNM) (Marx, 1969) sólido, a $25 \pm 1^{\circ} \mathrm{C}$, com repicagens a cada dois meses. Neste trabalho, os isolados fúngicos foram inicialmente cultivados em meio MNM sólido, em placas de Petri, em incubadora BOD a $25 \pm 1^{\circ} \mathrm{C}$. Após 30 dias, discos de $8 \mathrm{~mm}$ de diâmetro foram retirados das bordas das colônias e transferidos para erlenmeyers contendo $25 \mathrm{~mL}$ de MNM líquido. De cada isolado, foram preparados seis erlenmeyers com três discos de cultura cada. A incubação foi realizada sob as mesmas condições e, após 20 dias, o conteúdo de dois erlenmeyers foi fragmentado em liquidificador em $200 \mathrm{~mL}$ de meio MNM, durante $5 \mathrm{~s}$, sob condições assépticas, e utilizado na produção do inoculante.

$\mathrm{O}$ inoculante foi produzido em uma mistura turfavermiculita-meio de cultura em frasco do tipo conserva com $900 \mathrm{~mL}$ de capacidade. Cada frasco continha $500 \mathrm{~mL}$ da mistura turfa-vermiculita (1:4, v:v), e $200 \mathrm{~mL}$ do meio MNM líquido. As tampas dos frascos apresentavam furos de $1 \mathrm{~cm}$ de diâmetro fechados com fita porosa tipo Micropore, a fim de assegurar a aeração da cultura e evitar contaminações. A mistura foi previamente esterilizada (autoclavagem a $120^{\circ} \mathrm{C}$ ) durante $60 \mathrm{~min}$, antes da adição do meio líquido, sendo, em seguida, novamente esterilizada durante $20 \mathrm{~min}$. Após a inoculação das suspensões micelianas, os frascos foram mantidos em BOD, nas mesmas condições de incubação, durante dois meses. Para cada isolado, foram produzidos quatro frascos de inoculante. 
Antes da germinação, as sementes de E. dunnii foram desinfectadas em etanol $70 \%$ durante 30 s e lavadas três vezes consecutivas com água destilada estéril. A germinação foi realizada em solução estéril de ácido bórico $(3 \mu \mathrm{M})$, glicose $\left(2 \mathrm{~g} \mathrm{~L}^{-1}\right)$ e sulfato de cálcio $(500 \mu \mathrm{M}), \mathrm{pH} 5,7$, durante três dias, sob agitação.

O substrato de plantio turfa-vermiculita (1:3, v:v) foi previamente esterilizado duas vezes consecutivas durante 60 minutos cada, com um intervalo de 24 horas. A esse substrato foi adicionada solução nutritiva contendo os seguintes elementos (mg por planta): K, 16; $\mathrm{Mn}, 0,15 ; \mathrm{Mg}, 3$; Zn, 0,0375; Cu, 0,125; Mo, 0,05; B, 0,05; e Fe, 0,375 (Alves et al., 2001). Foram testadas sete doses de fósforo $(0,0,25,0,50,1,00,2,00,4,00 \mathrm{e}$ $8,00 \mathrm{mg}$ por planta), na forma de $\mathrm{Ca}\left(\mathrm{H}_{2} \mathrm{PO}_{4}\right)_{2} \cdot \mathrm{H}_{2} \mathrm{O}$. Após a eliminação do excesso de umidade do substrato a $70 \pm 1^{\circ} \mathrm{C}$, durante 72 horas, foi adicionado o inoculante fúngico, previamente lavado em $1.000 \mathrm{~mL}$ de água destilada estéril, na proporção de 10\% (v/v) (Alves et al., 2001). O tratamento testemunha, não-inoculado, recebeu a mesma quantidade da mistura turfa-vermiculitaMNM esterilizada, produzida e submetida às mesmas condições de manutenção e lavagem que o inoculante de fECM.

O substrato foi distribuído em tubetes cônicos de PVC de $60 \mathrm{~mL}$, previamente desinfectados durante 12 horas em solução de hipoclorito de sódio a $1 \%$ e lavados em água destilada. Cada tubete recebeu quatro sementes de $E$. dunnii pré-germinadas e foi irrigado com água destilada. Para cada combinação fungo-dose de P foram preparadas quatro repetições. Após a inoculação e a semeadura, os tubetes foram colocados em bandejas de polipropileno e mantidos em casa de vegetação, sendo irrigados diariamente com água destilada. Os tratamentos foram distribuídos num esquema fatorial, num delineamento completamente casualizado. Quatro semanas após o plantio, foi feito o desbaste, deixandose uma planta por tubete. A aplicação de N, na dosagem de $35 \mathrm{mg}$ por planta, foi parcelada em cinco vezes, a cada duas semanas a partir da segunda semana posterior ao plantio.

Após quatro meses, as plantas foram retiradas dos tubetes e analisadas quanto à colonização radicular, altura e diâmetro do caule, comprimento radicular total e colonizado, matéria seca, relação massa de matéria seca de raiz:massa de matéria seca da parte aérea (relação raiz:parte aérea) e conteúdo de $\mathrm{P}$ da parte aérea. A determinação da colonização radicular foi feita pela técnica das intersecções de Giovanetti \& Mosse (1980), modificada por Brundrett et al. (1996), utilizando-se amostras de peso $0,1 \mathrm{~g}$ de raízes frescas. De acordo com essa técnica, é possível estabelecer o comprimento do sistema radicular em função do número de pontos obtidos nas intersecções. O conteúdo de $\mathrm{P}$ foi determinado de acordo com Tedesco et al. (1995). Os dados foram submetidos à análise de variância e as médias comparadas pelo teste de Tukey a 5\% de probabilidade, utilizando-se o programa estatístico Statgraphic Plus (Manugistics).

\section{Resultados e Discussão}

A inoculação com fECM e os diferentes níveis de $\mathrm{P}$, considerados isoladamente, promoveram diferenças significativas na colonização radicular, absorção de $\mathrm{P}$, altura e diâmetro de plantas e massa de matéria seca.

O comprimento das raízes colonizadas variou com o isolado fúngico inoculado e com as doses de $\mathrm{P}$ aplicadas, não tendo sido observada interação entre esses dois fatores. Os isolados UFSC-Ch163 e UFSC-Pt116 promoveram maior comprimento de raiz colonizada em comparação com os tratamentos testemunha e UFSC-Hg93 (Tabela 1). Houve um descréscimo na colonização radicular com o aumento da dose de $\mathrm{P}$ aplicada ao substrato. As doses de 0,50 e 1,00 mg de P por planta produziram maior colonização que a dose de $8,00 \mathrm{mg}$ de $\mathrm{P}$ por planta, mas não diferiram significativamente das doses de 0, 2,00 e 4,00 mg de P por planta. Na dose de $8,00 \mathrm{mg}$ de $\mathrm{P}$ por planta não foi possível observar colonização micorrízica em qualquer dos tratamentos. Na maior parte dos casos, a colonização foi inibida a partir da dose de 4,00 mg de P por planta. Apenas as plantas com o isolado UFSC-Pt116 apresentaram colonização nessa dose de fósforo (Tabela 1).

Apesar de a porcentagem de colonização micorrízica obtida neste trabalho ter sido inferior à obtida por Bougher et al. (1990) e Alves et al. (2001), demonstrouse que, mesmo com uma baixa colonização, os isolados UFSC-Pt116 e UFSC-Ch163 foram capazes de promover benefícios às plantas, com um grau significativo de eficiência. Também é possível que diferenças nas técnicas de avaliação da colonização radicular possam ser responsáveis pelas diferenças de intensidade de colonização relatadas pelos diferentes autores.

As plantas submetidas à inoculação do isolado UFSC-Ch163 apresentaram altura superior à das plantas do tratamento testemunha e dos tratamentos INRA-Cou e UFSC-Hg93, mas não diferiram das submetidas à inoculação do isolado UFSC-Pt116 (Tabela 1). 
As maiores doses de $\mathrm{P}(2,00,4,00$ e $8,00 \mathrm{mg}$ de $\mathrm{P}$ por planta) promoveram maior altura de plantas. $\mathrm{O}$ diâmetro do caule aumentou com o aumento da dose de P, tendo sido observado um efeito favorável dos isolados UFSC-Ch163 e UFSC-Pt116 (Tabela 1), cujas plantas apresentaram diâmetro de caule maior as colonizadas pelo isolado UFSC-Hg93. No caso do tratamento UFSC-Ch163, a diferença também ocorreu em relação ao isolado INRA-Cou, mas não em relação às plantas testemunhas sem inoculação.

Os isolados UFSC-Pt116 e UFSC-Ch163 promoveram os maiores valores de massa de matéria seca
(Tabela 2). A massa de matéria seca total nas plantas com inoculação desses fungos foi $23 \%$ e $28 \%$ maior que a das plantas do tratamento testemunha, sem inoculação, respectivamente. $\mathrm{O}$ aumento da massa de matéria seca de plantas promovido pela inoculação desses dois isolados é um reflexo da maior biomassa produzida pela parte aérea e pelo sistema radicular das plantas desses tratamentos. A massa de matéria seca da parte aérea das plantas com inoculação dos isolados UFSC-Ch163 e UFSC-Pt116 foi cerca de, respectivamente, $27 \%$ e $24 \%$ maior que aquela produzida pelas plantas do tratamento testemunha. A matéria seca do

Tabela 1. Efeito da inoculação de isolados fúngicos ectomicorrízicos na colonização radicular, comprimento radicular, altura e diâmetro de mudas de Eucalyptus dunnii sob diferentes doses de P, em casa de vegetação. Médias de quatro repetições ${ }^{(1)}$.

\begin{tabular}{|c|c|c|c|c|c|c|}
\hline $\begin{array}{c}\text { P aplicado } \\
\text { (mg por planta) }\end{array}$ & Testemunha & INRA-Cou & UFSC-Hg93 & UFSC-Pt116 & UFSC-Ch163 & Média \\
\hline & \multicolumn{6}{|c|}{ Comprimento radicular colonizado (cm por planta) } \\
\hline 0,00 & 0 & 312 & 8 & 271 & 47 & $128 \mathrm{ab}$ \\
\hline 0,25 & 0 & 183 & 39 & 612 & 243 & $215 \mathrm{ab}$ \\
\hline 0,50 & 0 & 70 & 93 & 403 & 718 & $257 \mathrm{a}$ \\
\hline 1,00 & 0 & 241 & 35 & 292 & 670 & $247 \mathrm{a}$ \\
\hline 2,00 & 0 & 226 & 81 & 377 & 186 & $174 \mathrm{ab}$ \\
\hline 4,00 & 0 & 0 & 0 & 95 & 0 & $19 \mathrm{ab}$ \\
\hline 8,00 & 0 & 0 & 0 & 0 & 0 & $\mathrm{Ob}$ \\
\hline \multirow[t]{2}{*}{ Média } & $0 \mathrm{~b}$ & $147 \mathrm{ab}$ & $37 \mathrm{~b}$ & $293 a$ & $266 \mathrm{a}$ & \\
\hline & \multicolumn{6}{|c|}{ Comprimento radicular (m por planta) } \\
\hline 0,00 & 43 & 46 & 28 & 63 & 80 & $52 \mathrm{~d}$ \\
\hline 0,25 & 46 & 58 & 46 & 109 & 81 & $68 \mathrm{~cd}$ \\
\hline 0,50 & 63 & 64 & 60 & 102 & 138 & $85 \mathrm{~cd}$ \\
\hline 1,00 & 62 & 88 & 78 & 83 & 142 & $91 \mathrm{bc}$ \\
\hline 2,00 & 109 & 118 & 118 & 136 & 153 & $127 \mathrm{ab}$ \\
\hline 4,00 & 113 & 120 & 133 & 125 & 138 & $126 a b$ \\
\hline 8,00 & 128 & 118 & 122 & 157 & 139 & $133 a$ \\
\hline \multirow[t]{2}{*}{ Média } & $81 \mathrm{c}$ & $87 \mathrm{bc}$ & $84 b c$ & $111 \mathrm{ab}$ & $124 a$ & \\
\hline & \multicolumn{6}{|c|}{ Altura do caule (cm por planta) } \\
\hline 0,00 & 18,7 & 17,7 & 20,1 & 20,0 & 25,7 & $20,5 \mathrm{c}$ \\
\hline 0,25 & 18,6 & 22,6 & 21,6 & 24,1 & 23,9 & $22,2 \mathrm{c}$ \\
\hline 0,50 & 23,6 & 21,0 & 22,3 & 24,6 & 25,0 & $23,3 b c$ \\
\hline 1,00 & 26,5 & 23,1 & 25,9 & 25,5 & 29,3 & $26,1 \mathrm{ab}$ \\
\hline 2,00 & 24,8 & 27,6 & 25,2 & 28,8 & 33,8 & $28,0 \mathrm{a}$ \\
\hline 4,00 & 29,2 & 28,1 & 26,6 & 31,1 & 29,0 & $28,8 \mathrm{a}$ \\
\hline 8,00 & 23,6 & 26,6 & 31,3 & 28,1 & 30,4 & $28,0 \mathrm{a}$ \\
\hline \multirow[t]{2}{*}{ Média } & $23,6 b$ & $23,8 \mathrm{~b}$ & $24,7 b$ & $26,0 \mathrm{ab}$ & $28,1 \mathrm{a}$ & \\
\hline & \multicolumn{6}{|c|}{ Diâmetro do caule (cm por planta) } \\
\hline 0,00 & 0,24 & 0,27 & 0,21 & 0,28 & 0,37 & $0,27 \mathrm{~d}$ \\
\hline 0,25 & 0,28 & 0,29 & 0,28 & 0,29 & 0,36 & $0,30 \mathrm{~d}$ \\
\hline 0,50 & 0,36 & 0,28 & 0,30 & 0,39 & 0,35 & $0,34 \mathrm{c}$ \\
\hline 1,00 & 0,36 & 0,41 & 0,34 & 0,36 & 0,39 & $0,37 b c$ \\
\hline 2,00 & 0,44 & 0,40 & 0,36 & 0,45 & 0,39 & $0,41 \mathrm{ab}$ \\
\hline 4,00 & 0,40 & 0,41 & 0,41 & 0,44 & 0,45 & $0,42 \mathrm{a}$ \\
\hline 8,00 & 0,44 & 0,42 & 0,40 & 0,45 & 0,42 & $0,43 \mathrm{a}$ \\
\hline Média & $0,36 \mathrm{ab}$ & $0,35 b c$ & $0,33 c$ & $0,38 \mathrm{ab}$ & $0,39 a$ & \\
\hline
\end{tabular}

${ }^{(1)}$ Médias seguidas pela mesma letra, na linha ou na coluna, não diferem significativamente entre si pelo teste de Tukey a $5 \%$ de probabilidade. 
sistema radicular também foi $30 \%$ e $38 \%$ maior que a da testemunha nas plantas com inoculação dos isolados UFSC-Ch163 e UFSC-Pt116. As plantas com inoculação dos dois outros isolados não apresentaram diferenças significativas em relação à testemunha quanto a esses parâmetros.

A relação raiz:parte aérea não foi influenciada pela inoculação dos isolados fúngicos ectomicorrízicos (Tabela 2). Entretanto, houve efeito significativo da dose de P aplicada sobre este parâmetro. A relação foi maior nas plantas dos tratamentos que receberam as menores doses de $\mathrm{P}$, indicando a necessidade de um sistema radicular mais ramificado a fim de melhor explorar os recursos limitantes do substrato. A ausência de efeito da inoculação micorrízica sobre a relação raiz:parte aérea tem sido observada por outros autores (Bougher et al., 1990; Alves et al., 2001). Os aumentos simultâneos da matéria seca radicular e da matéria seca da parte aérea promovidos pela inoculação não se refletem na relação entre essas duas variáveis. Marx \& Ruehle (1991) indicam que a massa de matéria seca é o mais sensível indicador de respostas das mudas aos diferentes tratamentos, juntamente com o comprimento radicular. Nesses dois casos, os resultados demonstram

Tabela 2. Efeito da inoculação de isolados fúngicos ectomicorrízicos na produção de matéria seca e na acumulação de P na parte aérea de mudas de Eucalyptus dunnii sob diferentes doses de P, em casa de vegetação. Médias de quatro repetições ${ }^{(1)}$.

\begin{tabular}{|c|c|c|c|c|c|c|}
\hline $\begin{array}{c}\text { P aplicado } \\
\text { (mg por planta) }\end{array}$ & Testemunha & INRA-Cou & UFSC-Hg93 & UFSC-Pt116 & UFSC-Ch163 & Média \\
\hline & \multicolumn{6}{|c|}{ Massa de matéria seca da parte aérea (g por planta) } \\
\hline 0,00 & 0,41 & 0,64 & 0,49 & 0,86 & 1,11 & $0,70 \mathrm{~d}$ \\
\hline 0,25 & 0,60 & 0,73 & 0,56 & 0,93 & 1,21 & $0,81 \mathrm{~d}$ \\
\hline 0,50 & 1,08 & 1,01 & 0,96 & 1,67 & 1,61 & $1,27 \mathrm{c}$ \\
\hline 1,00 & 0,92 & 1,83 & 1,00 & 1,60 & 1,85 & $1,44 \mathrm{c}$ \\
\hline 2,00 & 2,46 & 2,54 & 2,31 & 2,78 & 2,65 & $2,55 b$ \\
\hline 4,00 & 2,84 & 2,84 & 2,79 & 2,92 & 2,89 & $2,86 \mathrm{ab}$ \\
\hline 8,00 & 3,03 & 2,87 & 3,19 & 3,28 & 3,12 & $3,10 \mathrm{a}$ \\
\hline \multirow[t]{2}{*}{ Média } & $1,62 b$ & $1,78 \mathrm{ab}$ & $1,61 \mathrm{~b}$ & $2,01 \mathrm{a}$ & $2,06 a$ & \\
\hline & \multicolumn{6}{|c|}{ Massa de matéria seca de raiz (g por planta) } \\
\hline 0,00 & 0,19 & 0,25 & 0,17 & 0,35 & 0,43 & $0,28 \mathrm{c}$ \\
\hline 0,25 & 0,21 & 0,30 & 0,22 & 0,60 & 0,45 & $0,35 \mathrm{bc}$ \\
\hline 0,50 & 0,39 & 0,26 & 0,33 & 0,66 & 0,49 & $0,43 b$ \\
\hline 1,00 & 0,35 & 0,50 & 0,40 & 0,52 & 0,60 & $0,47 b$ \\
\hline 2,00 & 0,64 & 0,64 & 0,59 & 0,78 & 0,63 & $0,65 \mathrm{a}$ \\
\hline 4,00 & 0,67 & 0,70 & 0,66 & 0,71 & 0,83 & $0,71 \mathrm{a}$ \\
\hline 8,00 & 0,78 & 0,69 & 0,70 & 0,83 & 0,77 & $0,75 \mathrm{a}$ \\
\hline \multirow[t]{2}{*}{ Média } & $0,46 \mathrm{~b}$ & $0,48 \mathrm{~b}$ & $0,44 \mathrm{~b}$ & $0,64 a$ & $0,60 \mathrm{a}$ & \\
\hline & \multicolumn{6}{|c|}{ Relação raiz:parte aérea } \\
\hline 0,00 & 0,49 & 0,48 & $0,47^{3}$ & 0,46 & 0,40 & $0,46 \mathrm{a}$ \\
\hline 0,25 & 0,43 & 0,42 & 0,41 & 0,67 & 0,39 & $0,47 \mathrm{a}$ \\
\hline 0,50 & 0,37 & 0,28 & 0,48 & 0,39 & 0,38 & $0,38 \mathrm{a}$ \\
\hline 1,00 & 0,40 & 0,29 & 0,43 & 0,35 & 0,34 & $0,36 a b$ \\
\hline 2,00 & 0,27 & 0,25 & 0,25 & 0,28 & 0,24 & $0,26 b$ \\
\hline 4,00 & 0,24 & 0,25 & 0,24 & 0,24 & 0,29 & $0,25 b$ \\
\hline 8,00 & 0,26 & 0,24 & 0,22 & 0,28 & 0,25 & $0,25 b$ \\
\hline \multirow[t]{2}{*}{ Média } & $0,35 \mathrm{a}$ & $0,32 \mathrm{a}$ & $0,36 \mathrm{a}$ & $0,38 \mathrm{a}$ & $0,33 \mathrm{a}$ & \\
\hline & \multicolumn{6}{|c|}{ Acumulação de P na parte aérea ( $\mu$ g por planta) } \\
\hline 0,00 & 17 & 37 & 35 & 51 & 92 & $46 e$ \\
\hline 0,25 & 35 & 56 & 36 & 62 & 117 & $61 \mathrm{de}$ \\
\hline 0,50 & 86 & 109 & 57 & 128 & 106 & $97 d$ \\
\hline 1,00 & 63 & 163 & 58 & 117 & 119 & $104 d$ \\
\hline 2,00 & 169 & 240 & 164 & 276 & 247 & $219 \mathrm{c}$ \\
\hline 4,00 & 282 & 343 & 220 & 382 & 354 & $316 b$ \\
\hline 8,00 & 604 & 469 & 551 & 658 & 627 & $582 \mathrm{a}$ \\
\hline Média & $179 \mathrm{bc}$ & $202 \mathrm{ab}$ & $160 \mathrm{c}$ & $239 \mathrm{a}$ & $238 \mathrm{a}$ & \\
\hline
\end{tabular}


a superioridade dos isolados UFSC-Ch163 e UFSC-Pt116.

Quanto à absorção de $\mathrm{P}$, as plantas diferiram em função da inoculação dos quatro isolados fúngicos (Tabela 2). As plantas com inoculação dos isolados UFSC-Ch163 e UFSC-Pt116 apresentaram teor de P da parte aérea cerca de $30 \%$ maior que o das plantas sem inoculação. Nas plantas com inoculação do isolado INRA-Cou, o acúmulo de $\mathrm{P}$ na parte aérea não diferiu significativamente das plantas com inoculação dos dois isolados citados anteriormente, como também em relação às plantas testemunhas. Finalmente, o isolado UFSC-Hg93 apresentou um conteúdo de $\mathrm{P}$ inferior em relação às plantas dos demais tratamentos de inoculação (Tabela 2).

Os isolados UFSC-Ch163 e UFSC-Pt116 foram aqueles que mais se destacaram. O incremento proporcionado pelos tratamentos de inoculação em relação aos valores obtidos nas plantas sem inoculação confirma a eficiência desses dois isolados em relação ao INRACou e UFSC-Hg93. Esse aumento pode ter resultado de vários mecanismos proporcionados pela colonização micorrízica. Entre eles destacam-se o aumento da área de absorção, devido à maior ramificação das raízes e ao micélio externo, e o efeito solubilizador dos fECM sobre fosfatos orgânicos presentes na turfa do substrato de plantio. Griffiths \& Caldwell (1992) citam a produção de fosfatases por fECM, atuando na mineralização do $P$ orgânico do solo. Assim, não somente houve um melhor aproveitamento do $\mathrm{P}$ adicionado, como pode ter havido a utilização do P orgânico presente na turfa, o que explicaria o efeito positivo dos fECM nas plantas sem $P$ aplicado (Tabela 2). Nos tratamentos sem $P$, as plantas sem inoculação apresentaram $17 \mu \mathrm{g}$ de $\mathrm{P}$ por planta, ao passo que as plantas com inoculação dos isolados UFSC-Ch163 e UFSC-Pt116 apresentaram 92 e $51 \mu \mathrm{g}$ de $\mathrm{P}$ por planta, respectivamente.

Na maioria dos parâmetros avaliados, as diferenças entre os isolados, ou entre estes e a testemunha, tenderam a ser maiores nas menores doses de $\mathrm{P}$, indicando uma inibição do efeito micorrízico em condições de alta fertilidade. Esse comportamento, embora já observado por outros autores com diferentes combinações de espécies vegetais e isolados de fungos ectomicorrízicos (Heinrich et al., 1988; Bougher et al., 1990; Soares et al., 1990), representa um dado importante nos estudos subseqüentes de seleção dos isolados para $E$. dunnii. Os resultados indicam que doses intermediárias de $\mathrm{P}$, da ordem de 0,50 a 1,00 mg por planta, seriam mais ade- quadas para compatibilizar uma colonização radicular significativa com um bom crescimento das mudas de E. dunnii.

Embora a colonização radicular pelo isolado UFSC-Ch163 não tenha sido detectada a partir da dose de 4,00 mg de P por planta, ainda foi possível observar efeitos positivos desse fungo nas plantas desse tratamento. As razões para esse resultado não estão claras, embora isso possa ser conseqüência da atividade do fungo na rizosfera mesmo que a simbiose não tenha se estabelecido em níveis detectáveis. Resultados semelhantes foram obtidos por Heinrich et al. (1988), em estudo com Eucalyptus pilularis e o fungo ectomicorrízico Pisolithus tinctorius, e mais recentemente por Narloch (2002) em estudos com Pinus taeda e vários isolados fúngicos ectomicorrízicos.

Os isolados UFSC-Pt116 e UFSC-Ch163 foram superiores em relação aos dois outros no que concerne à colonização e à promoção do crescimento das plantas de E. dunnii. Esses isolados são originários de plantações dessa espécie localizadas no Estado de Santa Catarina, demonstrando que os solos dessas plantações possuem potencial para proporcionar fungos eficientes que podem ser usados em programas de inoculação locais.

Fato inédito neste trabalho reside na utilização do isolado de Chondrogaster angustisporus (UFSC-Ch163), uma espécie nova descrita em plantações florestais do Estado de Santa Catarina (Giachini et al., 2000). Embora Brundrett et al. (1996) tenham observado a ocorrência de representantes do gênero Chondrogaster em plantações de eucalipto na Austrália, e sugerido tratarse de uma relação ectomicorrízica, este é o primeiro relato da inoculação de um fungo desse gênero em plantas, confirmando sua natureza micorrízica sugerida por esses autores.

\section{Conclusões}

1. A inoculação em mudas de Eucalyptus dunnii dos isolados fúngicos ectomicorrízicos UFSC-Ch163 (Chondrogaster angustisporus) e UFSC-Pt116 (Pisolithus microcarpus) aumenta o comprimento radicular, a absorção de fósforo e a produção de matéria seca.

2. A colonização ectomicorrízica é prejudicada pelo aumento do teor de fósforo aplicado ao substrato de plantio. 
3. A eficiência dos isolados tende a ser maior nas menores doses de fósforo aplicado.

\section{Agradecimentos}

À empresa florestal Rigesa, Celulose, Papel e Embalagens Ltda., Três Barras, SC, pelo fornecimento das sementes de Eucalyptus dunnii; aos colegas do Laboratório de Ectomicorrizas da UFSC, pela ajuda na coleta dos dados, pelas críticas e pelas sugestões.

\section{Referências}

ALVES, J.R.; SOUZA, O.; PODLECH, P.A.S.; GIACHINI, A.J.; OLIVEIRA, V.L. Efeito de inoculante ectomicorrízico produzido por fermentação semi-sólida no crescimento de Eucalyptus dunnii Maiden. Pesquisa Agropecuária Brasileira, v.36, p.307-313, 2001.

BOUGHER, N.L.; GROVE, T.S.; MALAJCZUK, N. Growth and phosphorus acquisition of kar ri (Eucalyptus diversicolor B. Muell.) seedlings inoculated with ectomycorrhizal fungi in relation to phosphorus supply. New Phytologist, v.114, p.77-85, 1990.

BRUNDRETT, M.; BOUGHER, N.L.; DELL, B.; GROVE, T.; MALAJCZUK, N. Working with mycorrhizas in forestry and agriculture. Canberra: Australian Centre for International Agricultural Research, 1996. 374p.

GARBAYE, J. Utilisation des mycorhizes en sylviculture. In: STRULLU, D.G. (Ed.). Les mycorhizes des arbres et plantes cultivées. Paris: Lavoisier, 1990. p.197-250.

GIACHINI, A.J.; OLIVEIRA, V.L.; CASTELLANO, M.A.; TRAPPE, J.M. Ectomycorrhizal fungi in Eucalyptus and Pinus plantations in southern Brazil. Mycologia, v.92, p.1166-1177, 2000.

GIOVANETTI, M.G.; MOSSE, B. An evaluation of techniques for measuring vesicular-arbuscular mycorrhizal infection in roots. New Phytologist, v.84, p.489-500, 1980.

GLOWA, K.R.; AROCENA, J.M.; MASSICOTTE, H.B. Extraction of potassium and/or magnesium from selected soil minerals by Piloderma. Geomicrobiology Journal, v.20, p.99-111, 2003.

GRIFFITHS, R.P.; CALDWELL, B.A. Mycorrhizal mat communities in forest soils. In: READ, D.J.; LEWIS, D.H.; FITTER, A.H.; ALEXANDER, I.J. (Ed.). Mycorrhizas in ecosystems. Wallingford: CAB International, 1992. p.98-105.

HASELWANDTER, K.; BOWEN, G.D. Mycorrhizal relations in trees for agroforestry and land rehabilitation. Forest Ecology and Management, v.81, p.1-17, 1996.

HEINRICH, P.A.; MULLIGAN, D.R.; PATRICK, J.W. The effect of ectomycorrhizas on the phosphorus and dry weight acquisition of Eucalyptus seedlings. Plant and Soil, v.109, p.147-149, 1988.

MARX, D.H. The influence of ectotrophic mycorrhizal fungi on the resistance of pine roots to pathogenic fungi and soil bactéria - I: antagonism of mycorrhizal fungi to root pathogenic fungi and soil bacteria. Phytopathology, v.59, p.153-163, 1969.

MARX, D.H.; BRYAN, W.C.; CORDELL, C.E. Survival and growth of pine seedlings with Pisolithus ectomycorrhizae after two years on reforestation sites in North Caroline and Florida. Forest Science, v.23, p.363-373, 1977.

MARX, D.H.; CORDELL, C.E. The use of specific ectomycorrhizas to improve artificial forestation practices. In: WHIPPS, J.M.; LUMSDEN, R.D. (Ed.). Biotechnology of fungi for improving plant growth. New York: Cambridge University Press, 1989. p.1-25.

MARX, D.H.; RUEHLE, J.L. Ectomycorrhizae as biological tools in reclamation and revegetation of waste lands. In: MAHADEVAN, A.; RAMAN, N.; NATARAJAN, K. (Ed.). Mycorrhizae for green Asia. Madras: Centre for Advanced Studies in Botany; University of Madras, 1988. p.336-344.

MARX, D.H.; RUEHLE, J.L. Methods for studying nursery and field responses of trees to specific ectomycorrhiza. In: NORRIS, J.R.; READ, D.J.; VARMA, A.K. (Ed.). Methods in microbiology. London: Academic, 1991. p.383-411.

MIKOLA, P. Application of mycorrhizal symbiosis in forestry practice. In: MARKS, G.C.; KOZLOWSKI, T.T. (Ed.). Ectomycorrhizae. London: Academic, 1973. p.383-411.

NARLOCH, C. Interação microrganismos solubilizadores de fosfatos-fungos ectomicorrízicos e o crescimento de Pinus taeda $\mathbf{L}$. 2002. 153p. Dissertação (Mestrado) - Universidade Federal de Santa Catarina, Florianópolis.

NEWSHAM, K.K.; FITTER, A.H.; WATKINSON, A.R. Multifunctionality and biodiversity in arbuscular mycorrhizas. Tree, v.10, p.407-411, 1995.

ROSSI, M.J.; SOUZA, J.A.R.; OLIVEIRA, V.L. Inoculum production of the ectomycorrhizal fungus Pisolithus microcarpus in an airlift bioreactor. Applied Microbiology and Biotechnology, v.59, p.175-181, 2002.

SAWYER, N.A.; CHAMBERS, S.M.; CAIRNEY, J.W.G. Utilisation of inorganic and organic phosphorus sources by isolates of Amanita muscaria and Amanita species native to temperate eastern Australia. Australian Journal of Botany, v.51, p.151-158, 2003.

SMITH, S.E.; READ, D.J. Mycorrhizal symbiosis. London: Academic, 1997. 605p.

SOARES, I.; BORGES, A.C.; BARROS, N.F.; BELLEI, M.M. Níveis de fósforo na formação de ectomicorrizas em mudas de eucalipto. Revista Brasileira de Ciência do Solo, v.14, p.327-332, 1990.

TEDESCO, M.J.; GIANELLO, C.; BISSANI, C.A.; BOHNEN, H.; VOLKWEISS, S.J. Análise de solo, plantas e outros materiais. 2.ed. Porto Alegre: UFRGS, 1995. 174p.

VOZZO, J.A.; HACSKAYLO, E. Inoculation of Pinus caribaea with ectomycorrhizal fungi in Puerto Rico. Forest Science, v.17, p.239-241, 1971.

WILCOX, H.E. Mycorrhizal associations. In: NAKAS, J.P.; HAGEDORN, C. (Ed.). Biotechnology of plant-microbe interactions. New York: McGraw-Hill, 1990. p.227-255. 